\title{
Effects of Pomace Olive Oil on Postprandial Lipid Metabolism in HIV Patients
}

\author{
J.S. Perona ${ }^{1}$, F. Rivas-García ${ }^{2}$, M. Aguilar ${ }^{3}$, J.L. Prada ${ }^{3}$, A. Del Arco ${ }^{3}$, M. García ${ }^{4}$, V. Ruiz-Gutierrez ${ }^{1}$ \\ 1 Instituto de la Grasa (CSIC), Seville, Spain \\ 2 Ayuntamiento de Guadix, Granada, Spain \\ ${ }^{3}$ Hospital Costa del Sol, Marbella, Spain \\ ${ }^{4}$ Hospital Virgen del Rocío, Seville, Spain
}

Highly active antirretroviral therapy (HAART) has contributed to improving the quality of life and lifespan of human immunodeficiency virus (HIV)-infected patients. Side effects of HAART include impairments of lipid metabolism, which are related to an increase in the incidence of cardiovascular disease among these patients. Postprandial lipid metabolism is also impaired in HIV-patients, who show a delayed clearance of triglyceride-rich lipoproteins (TRL) and their remnants. Olive oil has shown a protective effect in healthy subjects by reducing postprandial triglyceridemia compared with other oleic acid (18:1 n-9)-rich dietary oils, and its minor components can modulate the expression of genes involved in hepatic TRL uptake and secretion. Pomace olive oil (POO) is particularly rich in minor components, such as sterols, tocopherols and terpenoids, with antioxidant, hypocholesterolemic and anti-inflammatory properties. The aim of the present study was to determine if $\mathrm{POO}$, compared with a refined olive oil (ROO, devoid of minor components), can improve postprandial triglyceride concentrations in serum and TRL of HIV-patients.

Thirteen volunteers under antirretroviral treatment received two identical meals, differing only in the lipid source: POO or ROO. After the intake of the meals, blood was collected every hour for the subsequent $7 \mathrm{~h}$. TRL (Sf $>400)$ were isolated by means of ultracentrifugation and triglyceride, cholesterol and apolipoprotein (apo) B concentrations in serum and TRL were analyzed using commercial enzymatic kits. The concentrations of apo CIl and apo E in TRL were determined by Western-Blotting.

Serum triglyceride concentrations were higher after the intake of POO at all time points, showing a higher area under the curve (AUC) value. In TRL, triglycerides peaked $1 \mathrm{~h}$ later after POO intake. The same delay was found for cholesterol concentrations in TRL after POO intake but the AUC was significantly lower than after ROO intake. Apo B concentration in TRL was significantly lower and apo Cll higher at all times points after POO intake. Differences in apo E concentrations were only significant at $4 \mathrm{~h}$.

In conclusion, the present results show a marked effect of POO on postprandial lipid metabolism in HIV-patients, affecting the lipid and apolipoprotein composition of TRL. Therefore, the minor component composition of dietary oils may have an important role in modulating postprandial triglyceridemia in these patients. 